\title{
THE ALGEBRAIC EHP SEQUENCE
}

\author{
BY \\ WILLIAM M. SINGER( ${ }^{1}$ )
}

\begin{abstract}
Let $A$ be the dual of the mod-2 Steenrod algebra. If $M, N$, are graded unstable $A$-comodules, one can define and compute the derived functors $\operatorname{Coext}_{A}(M, N)$ using unstable injective resolutions of $N$. Bousfield and Curtis have shown that these unstable Coext groups can be fit into a long exact "EHP sequence", an algebraic analogue of the EHP sequence of homotopy theory. Our object in the present paper is to study the relationship between the $E$, $H$, and $P$ homomorphisms and the composition pairing $\operatorname{Coext}_{A}(N, R) \otimes$ $\operatorname{Coext}_{A}(M, N) \rightarrow \operatorname{Coext}_{A}(M, R)$. Among our results is a formula that measures the failure of the composition product to commute.
\end{abstract}

0. Introduction. Let $A$ be the dual of the mod-2 Steenrod algebra. If $M, N$ are graded unstable $A$-comodules (definitions below) once can define and compute the derived functors $\operatorname{Coxt}_{A}^{s}(M, N)$ using unstable injective resolutions of $N$. These derived functors were introduced by Massey and Peterson in [9], where they were used to express the $E_{2}$-term of an unstable Adams spectral sequence. Subsequently, Bousfield and Curtis ([3], [5]) showed these unstable Coext groups can be fit into a long exact "EHP sequence", an algebraic analogue of the EHP sequence of homotopy theory.

Our object in the present paper is to study the relations between the $E, H$, and $\boldsymbol{P}$ homomorphisms and the composition pairing

$$
\operatorname{Coext}_{A}(N, R) \otimes \operatorname{Coext}_{A}(M, N) \rightarrow \operatorname{Coext}_{A}(M, R) .
$$

Our first result in this direction is, roughly, that the order in which two elements are composed does not matter if they are suspended sufficiently often. More precisely, let $S^{p}$ (" $p$-sphere") be the unique unstable $A$-comodule for which $\left(S^{p}\right)_{p}=Z_{2},\left(S^{p}\right)_{n}=0 \quad(n \neq p)$. If $\alpha \in \operatorname{Coext}_{A}\left(S^{p+k}, S^{p}\right), \beta \in \operatorname{Coext}_{A}\left(S^{q+l}, S^{q}\right)$, then

$$
E^{q} \alpha \cdot E^{p+k} \beta=E^{p} \beta \cdot E^{q+l} \alpha
$$

Received by the editors December 20, 1973 and, in revised form, March 11, 1974.

AMS (MOS) subject classifications (1970). Primary 55H15, 55G 10, 55E40; Secondary $18 \mathrm{H15}, 18 \mathrm{G} 10$.

Key words and phrases. Cohomology of the Steenrod algebra, unstable Adams spectral sequence.

(1) Research partially supported by NSF grant GP-38484. 
This appears as Proposition 3.5 below, and is proved by identifying both sides with a "smash product" $\alpha \Lambda^{\beta}$ in $\operatorname{Coext}_{A}\left(S^{p+q+k+l}, S^{p+q}\right)$. (0.1) is an algebraic analogue of a result in homotopy theory; compare Barratt and Hilton [2]. This result leads us to consider the element

$$
[\alpha, \beta] \equiv E^{q-1} \alpha \cdot E^{p+k-1} \beta-E^{p-1} \beta \cdot E^{q+l-1} \alpha
$$

in $\operatorname{Coext}_{A}\left(S^{p+q+k+l-1}, S^{p+q-1}\right)$. (0.1) implies that $[\alpha, \beta]$ lies in ker $E=$ im $P$, and in $\S 4$ we obtain the formula

$$
E^{q-1} \alpha \cdot E^{p+k-1} \beta-E^{p-1} \beta \cdot E^{q+l-1} \alpha=P E\left(H \alpha{ }_{\Lambda} H \beta\right)
$$

showing how the composition product fails to commute, one suspension below the "stable range". This formula also is an algebraic analogue of a result in homotopy theory; compare Toda [13] and Barratt [1]. In $\S 6$ we construct a specific injective resolution of $S^{p}$ as an unstable $A$-comodule. Our construction makes use of the $\Lambda$-algebra ([3], [4], [5]), and relies on a lemma, Proposition 5.1 below, which tells how the subspaces $\Lambda(p) \subset \Lambda$ behave under multiplication. This lemma shows that our resolutions are particularly effective for computing composition pairings. A rule for computing composition pairings is given by Proposition 6.6. These results are then applied to obtain formulas for the Hopf invariant of a composition: see Propositions 3.7, 6.7, and 6.8 below. Our formulas are analogues of results in homotopy theory: compare Hilton [7].

I would like to thank Peter Bousfield for many useful conversations about unstable derived functors.

1. Unstable $A$-comodules. In this section we collect elementary facts about unstable $A$-comodules, and complexes of unstable $A$-comodules.

We write $A=Z_{2}\left(\xi_{1}, \xi_{2}, \cdots\right)$ for the dual of the Steenrod algebra, with coproduct $\psi: A \rightarrow A \otimes A$ as given by Milnor [10]. Define an increasing sequence of vector subspaces $J(0) \subseteq J(1) \subseteq \cdots \subseteq A$ by setting

$$
J(n)=\operatorname{Span}\left\{\xi_{1}^{n_{1}} \cdots \xi_{l}^{n_{l}} / n_{1}+\cdots+n_{l} \leqslant n\right\} .
$$

Each $J(n)$ is a left coideal in $A$ (that is, $\psi(J(n)) \subseteq A \otimes J(n)$ ); in fact, the stronger statement:

$$
\psi(J(n)) \subseteq \sum_{k=0}^{\infty} \oplus\left(J(n+k) \otimes J(n)_{k}\right)
$$

is valid. A graded $A$-comodule $M$ with structure map $\rho: M \rightarrow A \otimes M$ is called unstable if $\rho(M) \subseteq \Sigma_{n=0}^{\infty} \bigoplus\left(J(n) \otimes M_{n}\right)$. By a map of unstable $A$-comodules we will mean a degree-preserving $A$-map. Unstable $A$-comodules and their maps form an abelian category that we denote $M_{\boldsymbol{A}}$. 
We remark that our definition of an unstable $A$-comodule is dual to the usual definition [11, p. 27] of an unstable module over the Steenrod algebra $A^{*}$. To see this we need only observe that $J(n) \subseteq A$ is the annihilator of the ideal $B(n) \subseteq A^{*}$, where $B(n)$ is the span of all $S q^{I}$ with excess $(I)$ exceeding $n$. The relationship between $J(n)$ and $B(n)$ is discussed, for example, in [8].

We claim that $M_{A}$ has enough injectives. In fact, if $N$ is any graded $Z_{2}$ module write $A \otimes N=\Sigma_{n=0}^{\infty} \bigoplus\left(J(n) \otimes N_{n}\right)$. We regard $A \otimes N$ as an $A$ comodule, with structure map $\psi \otimes N$. (1.1) implies that $A \otimes N$ is unstable. To any morphism $f: M \rightarrow A \underline{\otimes} N$ in $M_{A}$ we associate a map of graded $Z_{2}$ modules $f: M \rightarrow N$ by setting $\underline{f}=(\epsilon \otimes N) f$, where $\epsilon: A \rightarrow Z_{2}$ is the counit. Then $f$ is determined by $\underline{f}$; in fact, $f=(A \otimes \underline{f}) \rho$. From this remark it follows easily that $A \unrhd N$ is an injective in $M_{A}$; also that $M_{A}$ "has enough injectives". From now on we reserve the term "injective" to denote an $M_{A}$ object of the special form $A \underline{\otimes}$.

By a "complex" $I$ we will always mean a complex over $M_{A}$ concentrated in nonnegative degrees with differential of degree $+1: \cdots 0 \rightarrow I^{0} \rightarrow I^{1} \rightarrow$ $\cdots$. Chain maps and chain homotopies are over the category $M_{A}$. They may have either positive or negative homological degree, but must preserve the grading ("internal degree") of each component $A$-comodule. If $I$ is a complex we denote by $\eta: H^{0}(I) \rightarrow I^{0}$ the canonical inclusion. If $I=I^{0} \rightarrow I^{1} \rightarrow \cdots$ is a complex and $F: M_{A} \rightarrow M_{A}$ is a functor, we denote by $F I$ the complex $F I^{0} \rightarrow F I^{1} \rightarrow \cdots$.

We will need some results on extending chain maps and chain homotopies. In the following two lemmas we assume $J$ a complex, and $J^{\prime} \subseteq J$ a subcomplex for which the induced map $H^{*}\left(J^{\prime}\right) \rightarrow H^{*}(J)$ is an isomorphism. Suppose $I$ is a complex of injectives.

Lemma 1.1. Suppose $\varphi^{\prime}: J^{\prime} \rightarrow I$ a chain map (of arbitrary homological degree). Then there exists a chain map $\varphi: J \rightarrow I$ which extends $\varphi^{\prime}$.

LEMma 1.2. Suppose $\varphi: J \rightarrow I$ a chain map. Let $\varphi^{\prime}: J^{\prime} \rightarrow I$ be the restriction of $\varphi$, and suppose there exists a chain homotopy $L^{\prime}: J^{\prime} \rightarrow I$ of $\varphi^{\prime}$ with zero. Then there exists a chain homotopy $L: J \rightarrow I$ of $\varphi$ with zero, which extends $L^{\prime}$.

The proofs are easy.

Given $N \in M_{A}$ we write $I(N)$ for any complex of injectives satisfying $H^{0}(I(N))=N, H^{s}(I(N))=0(s>0): I(N)$ is an injective resolution of $N$. Then for any $M \in M_{A}$, $\operatorname{Coext}(M, N)$ is the homology of the complex $\operatorname{Hom}(M, I(N))$ where we have abbreviated $\operatorname{Coext}_{A}, \operatorname{Hom}_{A}$, by writing simply Coext and Hom. The composition pairing $\operatorname{Coext}^{s}(N, R) \otimes \operatorname{Coext}^{r}(M, N) \rightarrow$ 
$\operatorname{Coext}^{r+s}(M, R)$ is defined in the obvious way. If $\alpha \in \operatorname{Coext}(M, N)$ and $\beta \in$ $\operatorname{Coext}(N, R)$, we write $\beta \cdot \alpha$ for their composition. Note that since "Hom" denotes maps which preserve internal degree, $\operatorname{Coext}(M, N)$ has only one grading: the homological degree.

We must now define the smash product. Observe that since $A$ is a Hopf algebra, the tensor product over $Z_{2}$ of a pair of $A$-comodules is again an $A$ comodule. The relation $J(n) \cdot J\left(n^{\prime}\right) \subseteq J\left(n+n^{\prime}\right)$ implies that a tensor product of unstable comodules is itself unstable. (But a tensor product of injectives in $M_{A}$ is not injective in $M_{A}$ !)

Definition 1.3. Let $N, N^{\prime} \in M_{A}$ be given. By a chain level smash product we mean a chain map $D: I(N) \otimes I\left(N^{\prime}\right) \rightarrow I\left(N \otimes N^{\prime}\right)$ of homological degree zero, for which the induced map $H^{0}\left(I(N) \otimes I\left(N^{\prime}\right)\right) \rightarrow H^{0}\left(I\left(N \otimes N^{\prime}\right)\right)$ is the identity on $N \otimes N^{\prime}$.

Since $I(N) \otimes I\left(N^{\prime}\right)$ is acyclic in dimensions greater than zero and $I\left(N \otimes N^{\prime}\right)$ is a complex of injectives such a chain map indeed exists, and is unique up to chain homotopy. Now given $\alpha \in \operatorname{Coext}^{r}(M, N), \alpha^{\prime} \in \operatorname{Coext}^{r^{\prime}}\left(M^{\prime}, N^{\prime}\right)$, we define the smash product $\alpha_{\Lambda} \alpha^{\prime} \in \operatorname{Coext}^{r+r^{\prime}}\left(M \otimes M^{\prime}, N \otimes N^{\prime}\right)$ as follows: if $\varphi(\alpha): M \rightarrow I(N), \varphi\left(\alpha^{\prime}\right): M^{\prime} \rightarrow I\left(N^{\prime}\right)$ are cycles representing $\alpha, \alpha^{\prime}$; then $D\left(\varphi(\alpha) \otimes \varphi\left(\alpha^{\prime}\right)\right): M \otimes M^{\prime} \rightarrow I\left(N \otimes N^{\prime}\right)$ represents $\alpha_{\Lambda} \alpha^{\prime}$.

Smash and composition products are related by:

Proposition 1.4. If $\alpha \in \operatorname{Coext}(M, N), \beta \in \operatorname{Coext}(N, R), \alpha^{\prime} \in \operatorname{Coext}\left(M^{\prime}, N^{\prime}\right)$, $\beta^{\prime} \in \operatorname{Coext}\left(N^{\prime}, R^{\prime}\right)$, then $(\beta \cdot \alpha)_{\Lambda}\left(\beta^{\prime} \cdot \alpha^{\prime}\right)=\left(\beta_{\Lambda} \beta^{\prime}\right) \cdot\left(\alpha_{\Lambda} \alpha^{\prime}\right)$.

The proof is straightforward.

2. Suspension and loop functors. In this section we collect information about the suspension and loop functors on $M_{A}$. The proofs of many of our statements (actually, their duals) can be found scattered through [3], and [9]; so. we have often omitted proofs.

Given $M \in M_{A}$ define its suspension $S M \in M_{A}$ by setting $(S M)_{n+1}=$ $M_{n}(n \geqslant 0),(S M)_{0}=0$. The structure map $\rho: S M \rightarrow A \otimes S M$ is inherited from $M$. Clearly $S(M \otimes N)=S M \otimes N=M \otimes S N$. Observe that the suspension of an injective is not injective!

Given $M \in M_{A}$ define a map $\lambda: M \rightarrow M$ of $Z_{2}$ modules as follows. If $x \in M_{2 n+1}$ has odd degree then $\lambda x=0$; if $x \in M_{2 n}$ has even degree write $\rho(x)=\xi_{1}^{n} \otimes \lambda x+\Sigma_{I}\left(\xi^{I} \otimes y^{I}\right)$ where the $\xi^{I} \in A$ are monomials distinct from $\xi_{1}^{n}$, and $y^{I} \in M$. $\lambda$ halves degree. If $M$ is an injective then $\lambda: M \rightarrow M$ is onto. We record the obvious:

Lemma 2.1. If $\lambda: M \rightarrow M$ is monic and $M_{0}=0$, then $M=0$.

Given $M \in M_{A}$ define its "loops" $\Omega M \in M_{A}$ by setting $(\Omega M)_{n}=(\operatorname{ker} \lambda)_{n+1}$. 
The structure map is inherited from $M$. It is easy to check that $\Omega M$ is unstable. $\Omega: M_{A} \rightarrow M_{A}$ is a left exact functor; but it is not right exact.

Note that $\Omega S$ is the identity functor. We have further:

Proposition 2.2. Let $M, N \in M_{A}$ be given. The natural correspondence which to any $f: S M \rightarrow N$ assigns $\Omega f: M \rightarrow \Omega N$ is an isomorphism of sets $\operatorname{Hom}(S M, N) \rightarrow \operatorname{Hom}(M, \Omega N) .(S, \Omega)$ is an adjoint pair.

We next observe that there are obvious inclusions $i: \Omega M \otimes N \rightarrow \Omega(M \otimes N)$; $j: M \otimes \Omega N \rightarrow \Omega(M \otimes N)$. Write $k$ for the composition $S(\Omega M \otimes \Omega N)=$ $\Omega M \otimes S \Omega N \rightarrow \Omega M \otimes N$; where $S \Omega N \rightarrow N$ is the image of the identity $\Omega N \rightarrow \Omega N$ under the adjunction isomorphism. A similar construction gives a map $l: S(\Omega M \otimes \Omega N) \rightarrow M \otimes \Omega N$. Clearly, $i k=j l$. These remarks will be useful in $\S 4$.

Finally we discuss the relationship between the loop functor and complexes of injectives.

PROPOSITION 2.3. $\Omega$ carries injectives to injectives. In fact, $\Omega(A \unrhd N)=$ $A \otimes L N$, where $L N$ is the graded $Z_{2}$-module defined by $(L N)_{n}=N_{n+1}$.

Suppose now $I$ is a complex of injectives. Then the sequence of $Z_{2}$ complexes: $0 \rightarrow \Omega I \rightarrow I \rightarrow I \rightarrow 0$ is short exact, and gives rise to a long exact sequence in homology:

$$
\cdots \rightarrow H^{s}(\Omega I) \rightarrow H^{s}(I) \stackrel{\lambda}{\longrightarrow} H^{s}(I) \stackrel{\delta}{\longrightarrow} H^{s+1}(\Omega I) \rightarrow \cdots .
$$

Each term in this sequence is an $M_{A}$ object. Combining these observations with Lemma 2.1 gives:

Proposition 2.4. Suppose I a complex of injectives; suppose $s \geqslant 0$ an integer for which $\left(I^{s}\right)_{0}=0$ and $H^{s}(\Omega I)=0$. Then $H^{s}(I)=0$.

Now let $M \in M_{A}$ be given. Let $I(M)$ be any injective resolution of $M$. Write $\Omega^{(s)} M=H^{s}(\Omega I(M))$ for the sth homology group of the complex $\cdots$ $0 \rightarrow \Omega I^{0}(M) \rightarrow \Omega I^{1}(M) \rightarrow \cdots$. Thus, $\Omega^{(s)}$ is the sth right derived functor of $\Omega$. From (2.1) we deduce easily:

Proposition 2.5. $\Omega^{(s)}=0$ if $s>1$. Further: $\left(\Omega^{(1)} M\right)_{2 n-1}=(M / \lambda M)_{n}$ and $\left(\Omega^{(1)} M\right)_{2 n}=0$. The structure map $\rho: \Omega^{(1)} M \rightarrow A \otimes \Omega^{(1)} M$ is given by the following rule. Let $x \in M$ represent $\Omega^{(1)} x \in \Omega^{(1)} M$. Suppose $\rho(x)=$ $\Sigma a_{i} \otimes x_{i}$. Then $\rho\left(\Omega^{(1)} x\right)=\Sigma a_{i}^{2} \otimes \Omega^{(1)} x_{i}$.

3. Suspension and Hopf invariant. In this section we give invariant definitions of the suspension and the Hopf invariant, and derive their elementary properties. 
Write $\Omega^{p}, S^{p}$ for the $p$-fold iterates of the loop and suspension functors.

DEFinition 3.1. Let $N \in M_{A}$ be given. By a chain level $p$-fold suspension we mean a chain map $e^{(p)}: I(N) \rightarrow \Omega^{p} I\left(S^{p} N\right)$ of homological degree zero, for which the induced map $H^{0}(I(N)) \rightarrow H^{0}\left(\Omega^{p} I\left(S^{p} N\right)\right)$ is the identity on $N$. A chain level one-fold suspension will be called simply a chain level suspension, and written $e: I(N) \rightarrow \Omega I(S N)$.

Since $I(N)$ is acyclic in dimensions greater than zero and $\Omega^{p} I\left(S^{p} N\right)$ is a complex of injectives, a chain level $p$-fold suspension exists, and is unique up to homotopy. Now we can define the suspension homomorphism

$$
E: \operatorname{Coext}^{r}(M, N) \rightarrow \operatorname{Coext}^{r}(S M, S N) \text {. }
$$

If $\varphi(\alpha): M \rightarrow I(N)$ is a cycle representing $\alpha \in \operatorname{Coxt}^{r}(M, N)$ form the composition $e \cdot \varphi(\alpha): M \rightarrow \Omega I(S N)$. Its adjoint $S M \rightarrow I(S N)$ is a cycle representing $E \alpha$.

Equivalently we could have defined a chain level $p$-fold suspension to be a chain map $f^{(p)}: S^{p} I(N) \rightarrow I\left(S^{p} N\right)$ of homological degree zero, for which the

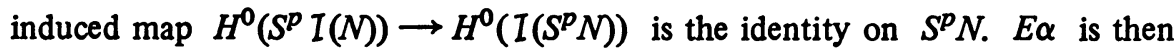
represented by the cycle $f \cdot S \varphi(\alpha): S M \rightarrow I(S N)$.

From the definitions we have easily:

Proposition 3.2. If $\alpha \in \operatorname{Coext}(M, N), \beta \in \operatorname{Coext}(N, R)$, then $E(\beta \cdot \alpha)=$ $E \beta \cdot E \alpha$ in $\operatorname{Coext}(S M, S R)$.

Denote by $S^{p}$ (" $p$-sphere") the unique $M_{A}$ object for which $\left(S^{p}\right)_{p}=Z_{2}$; $\left(S^{p}\right)_{n}=0 \quad(n \neq p)$. Let $i_{p} \in \operatorname{Coext}^{0}\left(S^{p}, S^{p}\right)$ be the identity map on $S^{p}$. Smash product and suspension are related by:

Proposition 3.3. Suppose $\alpha \in \operatorname{Coext}(M, N)$. Then $E^{p} \alpha=i_{p \Lambda} \alpha$ in $\operatorname{Coext}\left(S^{p} M, S^{p} N\right)$.

The proof is easy.

We now prove a sort of converse to Proposition 3.3 which will be needed in §4. Observe that $\eta \otimes I\left(S^{q}\right): S^{p} \otimes I\left(S^{q}\right) \rightarrow I\left(S^{p}\right) \otimes I\left(S^{q}\right)$ imbeds $S^{p} \otimes$ $I\left(S^{q}\right)$ as a subcomplex of $I\left(S^{p}\right) \otimes I\left(S^{q}\right)$. Similarly, we regard $I\left(S^{p}\right) \otimes S^{q}$ as a subcomplex of $I\left(S^{p}\right) \otimes I\left(S^{q}\right)$.

PROPOSITION 3.4. Suppose given arbitrary chain level $p$-fold and $q$-fold suspensions $f^{(p)}: S^{p} \otimes I\left(S^{q}\right) \rightarrow I\left(S^{p+q}\right) ; f^{(q)}: I\left(S^{p}\right) \otimes S^{q} \rightarrow I\left(S^{p+q}\right)$. Then there exists a chain level smash product $D: I\left(S^{p}\right) \otimes I\left(S^{q}\right) \rightarrow I\left(S^{p+q}\right)$ whose restriction to $S^{p} \otimes I\left(S^{q}\right)$ is $f^{(p)}$, and whose restriction to $I\left(S^{p}\right) \otimes S^{q}$ is $f^{(q)}$.

PROof. The inclusion $S^{p} \otimes I\left(S^{q}\right)+I\left(S^{p}\right) \otimes S^{q} \rightarrow I\left(S^{p}\right) \otimes I\left(S^{q}\right)$ induces isomorphisms in homology, so our proposition follows from Lemma 1.1. 
Proposition 3.5. Suppose $\alpha \in \operatorname{Coext}\left(S^{p+k}, S^{p}\right)$ and $\beta \in \operatorname{Coext}\left(S^{q+l}, S^{q}\right)$. Then

$$
E^{q} \alpha \cdot E^{p+k} \beta=E^{p} \beta \cdot E^{q+l} \alpha=\alpha_{\Lambda} \beta
$$

Proof. The proof imitates homotopy theory. (Compare [12, p. 25].) Combining Propositions 1.4 and 3.3 we obtain $E^{q} \alpha \cdot E^{p+k_{\beta}}=\left(\alpha_{\Lambda} i_{q}\right) \cdot$ $\left(i_{p+k \Lambda} \beta\right)=\left(\alpha \cdot i_{p+k}\right)_{\Lambda}\left(i_{q} \cdot \beta\right)=\alpha_{\Lambda} \beta$. The identity $E^{p} \beta \cdot E^{q+l_{\alpha}}=\alpha_{\Lambda} \beta$ is proved in the same way.

We turn now to the Hopf invariant.

DEFinition 3.6. Let $N \in M_{A}$ be given. By a chain level Hopf invariant we mean a chain map $h: \Omega I(N) \rightarrow I\left(\Omega^{(1)} N\right)$ of homological degree -1 , for which the induced map $H^{1}(\Omega I(N)) \rightarrow H^{0}\left(I\left(\Omega^{(1)} N\right)\right)$ is the identity on $\Omega^{(1)} N$.

Since $\Omega I(N)$ is acyclic in dimensions greater than one and $I\left(\Omega^{(1)} N\right)$ is a complex of injectives, such a chain map exists and is unique up to homotopy. Now we can define the Hopf invariant

$$
H: \operatorname{Coext}^{r}(S M, N) \rightarrow \operatorname{Coext}^{r-1}\left(M, \Omega^{(1)} N\right) .
$$

If $\varphi(\alpha): S M \rightarrow I(N)$ represents $\alpha \in \operatorname{Coext}^{r}(S M, N)$, then the composition $h \cdot \Omega \varphi(\alpha)$ represents $H \alpha$.

Proposition 3.7. If $\alpha \in \operatorname{Coext}^{r}(S N, R), \beta \in \operatorname{Coext}^{s}(M, N)$, then $H(\alpha \cdot E \beta)=(H \alpha) \cdot \beta$ in $\operatorname{Coext}^{r+s-1}\left(M, \Omega^{(1)} R\right)$.

Proof. Let $\varphi(\alpha): I(S N) \rightarrow I(R)$ be a chain map of homological degree $+r$ representing $\alpha$; let $\varphi(\beta): M \rightarrow I(N)$ be a cycle representing $\beta$. Our definitions imply that both $H(\alpha \cdot E \beta)$ and $H \alpha \cdot \beta$ are represented by the composition

$$
M \stackrel{\varphi(\beta)}{\longrightarrow} I(N) \stackrel{e}{\longrightarrow} \Omega I(S N) \stackrel{\Omega \varphi(\alpha)}{\longrightarrow} \Omega I(R) \stackrel{h}{\longrightarrow} I\left(\Omega^{(1)} R\right) .
$$

The next result about Hopf invariants will be needed in $\S 4$. Observe (Proposition 2.5) that $\Omega^{(1)} S^{n}=S^{2 n-1}$. Then $H^{2}\left(S\left(\Omega I\left(S^{p}\right) \otimes \Omega I\left(S^{q}\right)\right)\right)=$ $S^{2 p+2 q-1}$, and $S\left(\Omega I\left(S^{p}\right) \otimes \Omega I\left(S^{q}\right)\right)$ is acyclic in dimensions greater than two. Therefore there exists a chain map

$$
G: S\left(\Omega I\left(S^{p}\right) \otimes \Omega I\left(S^{q}\right)\right) \rightarrow I\left(S^{2 p+2 q-1}\right)
$$

of homological degree -2 , for which the induced map

$$
H^{2}\left(S\left(\Omega I\left(S^{p}\right) \otimes \Omega I\left(S^{q}\right)\right)\right) \rightarrow H^{0}\left(I\left(S^{2 p+2 q-1}\right)\right)
$$

is the identity on $S^{2 p+2 q-1}$. Such a $G$ is unique up to chain homotopy, and from this fact there follows easily:

Proposition 3.8. Let $\varphi(\alpha): S^{p+k} \rightarrow I\left(S^{p}\right) ; \varphi(\beta): S^{q+l} \rightarrow I\left(S^{q}\right)$ represent $\alpha \in \operatorname{Coext}\left(S^{p+k}, S^{p}\right) ; \beta \in \operatorname{Coext}\left(S^{q+l}, S^{q}\right)$ respectively. Then the composition 


$$
G \cdot(S(\Omega \varphi(\alpha) \otimes \Omega \varphi(\beta))): S^{p+q+k+l-1} \rightarrow I\left(S^{2 p+2 q-1}\right)
$$

represents $E\left(H \alpha_{\Lambda} H \beta\right)$ in $\operatorname{Coext}\left(S^{p+q+k+l-1}, S^{2 p+2 q-1}\right)$.

Finally, we anticipate a result of $\S 6$. There we will construct for each integer $p \geqslant 1$ a short exact sequence of injective resolutions:

$$
0 \rightarrow L\left(S^{p-1}\right) \stackrel{e}{\longrightarrow} \Omega L\left(S^{p}\right) \stackrel{h}{\longrightarrow} L\left(S^{2 p-1}\right) \rightarrow 0
$$

where $e$ is a chain level suspension and $h$ is a chain level Hopf invariant. From (3.1) we obtain immediately a long exact "EHP sequence" for any $k \geqslant 0$ :

$$
\begin{aligned}
\cdots & \rightarrow \operatorname{Coext}^{r}\left(S^{p+k-1}, S^{p-1}\right) \stackrel{E}{\longrightarrow} \operatorname{Coext}^{r}\left(S^{p+k}, S^{p}\right) \\
& \stackrel{H}{\longrightarrow} \operatorname{Coext}^{r-1}\left(S^{p+k-1}, S^{2 p-1}\right) \stackrel{P}{\longrightarrow} \operatorname{Coext}^{r+1}\left(S^{p+k-1}, S^{2 p-1}\right) \rightarrow \cdots
\end{aligned}
$$

where $P$ is the connecting morphism. It is identical with the EHP sequences of [3] and [5].

4. Noncommutativity of the composition product. Suppose given $\alpha \in$ $\operatorname{Coext}^{r}\left(S^{p+k}, S^{p}\right), \beta \in \operatorname{Coext}^{s}\left(S^{q+l}, S^{q}\right)$. Define the "commutator" $[\alpha, \beta]$ by setting: $[\alpha, \beta]=E^{q-1} \alpha \cdot E^{p+k-1} \beta-E^{p-1} \beta \cdot E^{q+l-1} \alpha$. Propositions 3.2 and 3.5 imply that $E[\alpha, \beta]=0$, so $[\alpha, \beta]$ must lie in the image of $P$. The main result of this section is:

THEOREM 4.1. $[\alpha, \beta]=P E\left(H \alpha{ }_{\Lambda} H \beta\right)$.

The proof requires some preliminary constructions.

First, we assume given a short exact sequence of injective resolutions

$$
0 \rightarrow L\left(S^{p+q-1}\right) \stackrel{e}{\longrightarrow} \Omega L\left(S^{p+q}\right) \stackrel{h}{\longrightarrow} L\left(S^{2 p+2 q-1}\right) \rightarrow 0
$$

where $e$ is a chain level suspension and $h$ is a chain level Hopf invariant. In this section we will not need to know any more about the complexes $L$ beyond the fact that they can fit into a short exact sequence of this form, so we have postponed their construction to $\S 6$.

Next, we need injective resolutions of $S^{p}$ and $S^{q}$. For uniformity of notation we choose $L\left(S^{p}\right)$ and $L\left(S^{q}\right)$, although the proof would go through with arbitrary choices.

Now choose cycles $\varphi(\alpha): S^{p+k} \rightarrow L\left(S^{p}\right), \varphi(\beta): S^{q+l} \rightarrow L\left(S^{q}\right)$ to represent $\alpha, \beta$ respectively. Choose and keep fixed throughout this section a chain level $(q-1)$-fold suspension $f^{(q-1)}: L\left(S^{p}\right) \otimes S^{q-1} \rightarrow L\left(S^{p+q-1}\right)$ and a chain level $(p-1)$-fold suspension $f^{(p-1)}: S^{p-1} \otimes L\left(S^{q}\right) \rightarrow L\left(S^{p+q-1}\right)$. Choose a chain map $\varphi\left(E^{q-1} \alpha\right): S^{p+k-1} \otimes L\left(S^{q}\right) \rightarrow L\left(S^{p+q-1}\right)$ of homological degree 
$+r$, in such a way that

$$
f^{(q-1)} \cdot\left(\varphi(\alpha) \otimes S^{q-1}\right)=\varphi\left(E^{q-1} \alpha\right) \cdot\left(S^{p+k-1} \otimes \eta\right)
$$

(recall $\eta: S^{q} \rightarrow L\left(S^{q}\right)$ is the canonical inclusion). Similary, choose a chain map $\varphi\left(E^{p-1} \beta\right): L\left(S^{p}\right) \otimes S^{q+l-1} \rightarrow L\left(S^{p+q-1}\right)$ of homological degree $+s$, in such a way that

$$
f^{(p-1)} \cdot\left(S^{p-1} \otimes \varphi(\beta)\right)=\varphi\left(E^{p-1} \beta\right) \cdot\left(\eta \otimes S^{q+l-1}\right) .
$$

The existence of such chain maps is assured by the fact that $S^{p+k-1} \otimes L\left(S^{q}\right)$ and $L\left(S^{p}\right) \otimes S^{q+l-1}$ are acyclic in dimensions greater than zero, while $L\left(S^{p+q-1}\right) \cdot$ is a complex of injectives.

Now choose and keep fixed throughout this section a chain level smash product $D: L\left(S^{p}\right) \otimes L\left(S^{q}\right) \rightarrow L\left(S^{p+q}\right)$ whose restriction to $L\left(S^{p}\right) \otimes S^{q}$ is the composition $f \cdot S f^{(q-1)}$ and whose restriction to $S^{p} \otimes L\left(S^{q}\right)$ is the composition $f \cdot S f^{(p-1)}$. Here we have written $f: S L\left(S^{p+q-1}\right) \rightarrow L\left(S^{p+q}\right)$ for the adjoint of $e: e=\Omega f$. The existence of such a smash product is given by Proposition 3.4.

The diagram (4.4) is the master diagram for this section. Solid arrows represent chain maps which have already been constructed. ( $i, j$ are as in \$2). All closed circuits formed by solid arrows are, as we shall see, at least homotopy commutative. Dotted arrows represent chain homotopies which will be constructed in the text.

LEMMA 4.2. The lower pentagon in (4.4) is homotopy commutative. That is, the chain maps $e \cdot \varphi\left(E^{q-1} \alpha\right)$ and $\Omega D \cdot i \cdot\left(\Omega \varphi(\alpha) \otimes L\left(S^{q}\right)\right)$ are homotopic.

Proof. It suffices to show that $e \cdot \varphi\left(E^{q-1} \alpha\right)$ and $\Omega D \cdot i \cdot(\Omega \varphi(\alpha) \otimes$ $L\left(S^{q}\right)$ ) become equal when composed on the right with the canonical inclusion

$$
S^{p+k-1} \otimes \eta: S^{p+k-1} \otimes S^{q} \rightarrow S^{p+k-1} \otimes L\left(S^{q}\right) .
$$

But this fact follows from (4.2), our construction of $D$, and the relation $\Omega f=e$.

The proof of Lemma 4.2 has shown more than its statement: we can choose a chain homotopy $K(\alpha): S^{p+k-1} \otimes L\left(S^{q}\right) \rightarrow \Omega L\left(S^{p+q}\right)$ between the two edges of the pentagon, in such a way that the composition $K(\alpha) \cdot\left(S^{p+k-1} \otimes \eta\right)$ is zero. We do this.

A similar argument shows that the upper pentagon in (4.4) is homotopy commutative. We choose a chain homotopy $K(\beta): L\left(S^{p}\right) \otimes S^{q+l-1} \rightarrow \Omega L\left(S^{p+q}\right)$ between the maps $e \cdot \varphi\left(E^{p-1} \beta\right)$ and $\Omega D \cdot j \cdot\left(L\left(S^{p}\right) \otimes \Omega \varphi(\beta)\right)$, in such a way that the composition $K(\beta) \cdot\left(\eta \otimes S^{q+l-1}\right)$ is zero. $K(\alpha), K(\beta)$ have homological degrees $r-1, s-1$ respectively. 


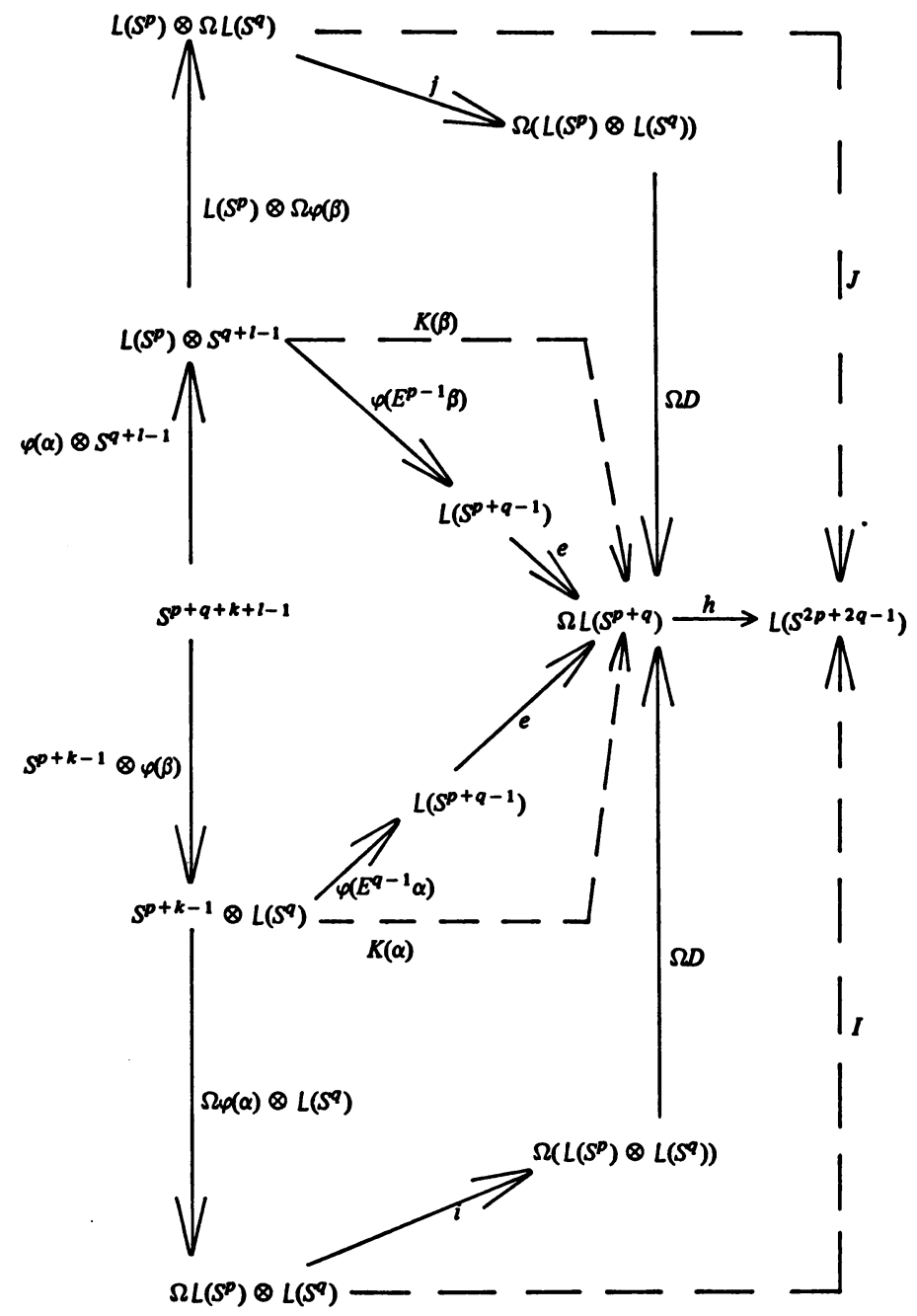

Next (see (4.4)) we observe that the map

$$
h \cdot\left[K(\alpha) \cdot\left(S^{p+k-1} \otimes \varphi(\beta)\right)-K(\beta) \cdot\left(\varphi(\alpha) \otimes S^{q+l-1}\right)\right]
$$

is a cycle in the complex $\operatorname{Hom}\left(S^{p+q+k+l-1}, L\left(S^{2 p+2 q-1}\right)\right)$. This follows immediately from the definitions of $K(\alpha)$ and $K(\beta)$, the relation $h e=0$, and the relation $i \cdot[\Omega \varphi(\alpha) \otimes \varphi(\beta)]=j \cdot[\varphi(\alpha) \otimes \Omega \varphi(\beta)]$. Let $\rho(\alpha, \beta)$ be the element of Coext ${ }^{r+s-2}\left(S^{p+q+k+l-1}, S^{2 p+2 q-1}\right)$ represented by the cycle (4.5).

LEMMA 4.3. $[\alpha, \beta]=P \rho(\alpha, \beta)$.

Proof. Reference to (4.4) is helpful. The lemma follows easily from the 
fact that $P$ is the connecting morphism associated with the short exact sequence (4.1), and the fact that $\varphi\left(E^{q-1} \alpha\right) \cdot\left(S^{p+k-1} \otimes \varphi(\beta)\right)$ is a cycle representing $E^{q-1} \alpha \cdot E^{p+k-1} \beta$, while $\varphi\left(E^{p-1} \beta\right) \cdot\left(\varphi(\alpha) \otimes S^{q+l-1}\right)$ is a cycle representing $E^{p-1} \beta \cdot E^{q+l-1} \alpha$.

To complete the proof of Theorem 4.1 we must identify $\rho(\alpha, \beta)$ with $E\left(H \alpha \alpha_{\Lambda} H \beta\right)$. The first step is:

LEMMA 4.4. The chain map $h \cdot \Omega D \cdot i: \Omega L\left(S^{p}\right) \otimes L\left(S^{q}\right) \rightarrow L\left(S^{2 p+2 q-1}\right)$ is homotopic to zero. In fact, one can choose a chain homotopy $I$ of $h \cdot \Omega D \cdot i$ with zero, in such a way that the composition $I \cdot\left(\Omega L\left(S^{p}\right) \otimes \eta\right): \Omega L\left(S^{p}\right) \otimes S^{q}$ $\rightarrow L\left(S^{2 p+2 q-1}\right)$ is zero.

Proof. Our construction of $D$ and the relations $\Omega f=e, h e=0$, show that the composition $h \cdot \Omega D \cdot i \cdot\left(\Omega L\left(S^{p}\right) \otimes \eta\right)$ is the zero map. Lemma 4.4 is therefore an application of Lemma 1.2, with

$$
\begin{aligned}
& J=\Omega L\left(S^{p}\right) \otimes L\left(S^{q}\right), \quad I=L\left(S^{2 p+2 q-1}\right), \\
& \varphi=h \cdot \Omega D \cdot i, \quad J^{\prime}=\Omega L\left(S^{p}\right) \otimes S^{q}, \varphi^{\prime}=0 .
\end{aligned}
$$

A similar argument shows that the chain map $h \cdot \Omega D \cdot j: L\left(S^{p}\right) \otimes \Omega L\left(S^{q}\right)$ $\rightarrow L\left(S^{2 p+2 q-1}\right)$ is homotopic to zero; and that we can choose a homotopy $J$ in such a way that the composition $J \cdot\left(\eta \otimes \Omega L\left(S^{q}\right)\right): S^{p} \otimes \Omega L\left(S^{q}\right) \rightarrow$ $L\left(S^{2 p+2 q-1}\right)$ is zero. The homotopies $I, J$ are shown in (4.4). Each has homological degree -2 .

Next we observe that each of the maps $h \cdot K(\alpha), I \cdot\left(\Omega \varphi(\alpha) \otimes L\left(S^{q}\right)\right)$ is a homotopy of the composition $h \cdot \Omega D \cdot i \cdot\left(\Omega \varphi(\alpha) \otimes L\left(S^{q}\right)\right)$ with zero. This suggests:

LEMmA 4.5. The homotopies $h \cdot K(\alpha)$ and $I \cdot\left(\Omega \varphi(\alpha) \otimes L\left(S^{q}\right)\right)$ are themselves homotopic.

Proof. To what has already been said we need only add that $S^{p+k-1} \otimes$ $L\left(S^{q}\right)$ is acyclic in dimensions greater than zero, $L\left(S^{2 p+2 q-1}\right)$ is a complex of injectives, and the compositions

$$
h \cdot K(\alpha) \cdot\left(S^{p+k-1} \otimes \eta\right) \text { and } I \cdot\left(\Omega \varphi(\alpha) \otimes L\left(S^{q}\right)\right) \cdot\left(S^{p+k-1} \otimes \eta\right)
$$

are equal maps from $S^{p+k-1} \otimes S^{q}$ into $L\left(S^{2 p+2 q-1}\right)$. In fact, we have chosen $K(\alpha)$ and $I$ in such a way that both of these compositions are zero!

A similar argument shows that the homotopies $h \cdot K(\beta)$ and $J \cdot\left(L\left(S^{p}\right) \otimes\right.$ $\Omega \varphi(\beta))$ are homotopic. Then, we can alter the cycle (4.5) by replacing $h \cdot K(\alpha)$ by $I \cdot\left(\Omega \varphi(x) \otimes L\left(S^{q}\right)\right)$, and $h \cdot K(\beta)$ by $J \cdot\left(L\left(S^{p}\right) \otimes \Omega \varphi(\beta)\right)$, without changing its homology class. But if the maps 
and

$$
k: S\left(\Omega L\left(S^{p}\right) \otimes \Omega L\left(S^{q}\right)\right) \rightarrow \Omega L\left(S^{p}\right) \otimes L\left(S^{q}\right)
$$

$$
l: S\left(\Omega L\left(S^{p}\right) \otimes \Omega L\left(S^{q}\right)\right) \rightarrow L\left(S^{p}\right) \otimes \Omega L\left(S^{q}\right)
$$

are as in $\$ 2$, we have

$$
k \cdot S(\Omega \varphi(\alpha) \otimes \Omega \varphi(\beta))=\Omega \varphi(\alpha) \otimes \varphi(\beta)
$$

and

$$
l \cdot S(\Omega \varphi(\alpha) \otimes \Omega \varphi(\beta))=\varphi(\alpha) \otimes \Omega \varphi(\beta)
$$

We conclude:

LEMma 4.6. $\rho(\alpha, \beta)$ in $\operatorname{Coext}^{r+s-2}\left(S^{p+q+k+l-1} S^{2 p+2 q-1}\right)$ is represented by the cycle: $(I k-J l) \cdot(S(\Omega \varphi(\alpha) \otimes \Omega \varphi(\beta)))$.

Combining Lemmas 4.3 and 4.6, we find that the proof of Theorem 4.1 requires only:

LEMMA 4.7. The cycle $(I k-J l) \cdot(S(\Omega \varphi(\alpha) \otimes \Omega \varphi(\beta)))$ represents $E\left(H \alpha \alpha_{\Lambda} H \beta\right)$.

Proof. The definitions of $I$ and $J$ and the relation $i k=j l$ imply that $I k-J: S\left(\Omega L\left(S^{p}\right) \otimes \Omega L\left(S^{q}\right)\right) \rightarrow L\left(S^{2 p+2 q-1}\right)$ is a chain map of homological degree - 2. In view of Proposition 3.8, we need only show that the map $H^{2}\left(S\left(\Omega L\left(S^{p}\right) \otimes \Omega L\left(S^{q}\right)\right)\right) \rightarrow H^{0}\left(L\left(S^{2 p+2 q-1}\right)\right)$ induced by $I k-J$ is the identity on $S^{2 p+2 q-1}$. To do this we anticipate $\S 6$ and introduce a minimum of specific information about the complexes $L\left(S^{n}\right)$ : namely $L^{0}\left(S^{n}\right)=A \otimes S^{n}$. Write $\sigma_{n} \in\left(S^{n}\right)_{n}$ for the nonzero class, and set $\sigma_{n}^{1}=d\left(\xi_{1}^{n} \otimes \sigma_{n}\right)$ in $L^{1}\left(\bar{S}^{n}\right)$. Then $\sigma_{n}^{1}$ represents an element of $\Omega L^{1}\left(S^{n}\right)$ which we denote $\Omega \sigma_{n}^{1}$. It is easy to see that $\Omega \sigma_{n}^{1}$ represents the nonzero class in $\Omega^{(1)} S^{n}=S^{2 n-1}$. Define $a_{I}$, $a_{J}$ in $Z_{2}$ by setting $I\left(\Omega \sigma_{p}^{1} \otimes \sigma_{p}^{1}\right)=a_{I} \sigma_{2 p+2 q-1}, J\left(\sigma_{p}^{1} \otimes \Omega \sigma_{q}^{1}\right)=a_{J} \sigma_{2 p+2 q-1}$. Since $I$ is a chain homotopy of $h \cdot \Omega D \cdot i$ with zero we must have

$$
a_{I} \sigma_{2 p+2 q-1}=I d\left(\Omega \sigma_{p}^{1} \otimes\left(\xi_{1}^{q} \otimes \sigma_{q}\right)\right)=h \cdot \Omega D\left(\Omega\left(\sigma_{p}^{1} \otimes\left(\xi_{1}^{q} \otimes \sigma_{q}\right)\right)\right) .
$$

Similarly $a_{J} \sigma_{2 p+2 q-1}=h \cdot \Omega D\left(\Omega\left(\left(\xi_{1}^{p} \otimes \sigma_{p}\right) \otimes \sigma_{q}^{1}\right)\right)$, so that

$$
\begin{aligned}
\left(a_{I}+a_{J}\right) \sigma_{2 p+2 q-1} & =h \cdot \Omega D \cdot d\left(\Omega\left(\xi_{1}^{p} \otimes \sigma_{p} \otimes \xi_{1}^{q} \otimes \sigma_{q}\right)\right) \\
& =h \cdot d\left(\Omega\left(D\left(\xi_{1}^{p} \otimes \sigma_{p} \otimes \xi_{1}^{q} \otimes \sigma_{q}\right)\right)\right) .
\end{aligned}
$$

But the definition of a chain level smash product forces $D\left(\xi_{1}^{p} \otimes \sigma_{p} \otimes \xi_{1}^{q} \otimes \sigma_{q}\right)=$ $\xi_{1}^{p+q} \sigma_{p+q}$, so we now have $\left(a_{I}+a_{J}\right) \sigma_{2 p+2 q-1}=h\left(\Omega \sigma_{p+q}^{1}\right)=\sigma_{2 p+2 q-1}$, so that $a_{I}+a_{J}=1$. Consequently,

$$
(I k-J t)\left(S\left(\Omega \sigma_{p}^{1} \otimes \Omega \sigma_{q}^{1}\right)\right)=I\left(\Omega \sigma_{p}^{1} \otimes \sigma_{q}^{1}\right)+J\left(\sigma_{p}^{1} \otimes \Omega \sigma_{q}^{1}\right)=\sigma_{2 p+2 q-1} .
$$


This completes the proof of Lemma 4.7, and with it the proof of Theorem 4.1.

5. Some computations with the $\Lambda$-algebra. In this section we present the results of some computations with the $\Lambda$-algebra. These results will be useful in constructing the complexes $L\left(S^{p}\right)$, and in deriving formulas for the Hopf invariant of a composition.

Recall from [3], [4], [5] that $\Lambda=\Sigma_{s \geqslant 0} \Sigma_{k \geqslant 0} \oplus \Lambda_{k}^{s}$ is a bigraded differential algebra generated by a unit 1 and elements $\lambda_{i} \in \Lambda_{i+1}^{1}$ for each $i \geqslant 0$. (Note that our definition of the grading is different from that of [3] and [4], where $\lambda_{i} \in \Lambda_{i}^{1}$.) The relations among the $\lambda_{i}$ 's and the definition of the differential $d_{\Lambda}: \Lambda \rightarrow \Lambda$ of bidegree $(1,0)$ are given in [3], [4]. We write $\Lambda(p) \subseteq$ $\Lambda$ for the subcomplex spanned by those admissibles $\lambda_{I}=\lambda_{i_{1}} \cdots \lambda_{i_{s}}$ for which $i_{1}<p$. Write $h_{p}: \Lambda(p) \rightarrow \Lambda(2 p-1)$ for the chain map of bidegree $(-1,-p)$ defined on admissibles by the rule $h_{p}\left(\lambda_{i_{1}} \cdots \lambda_{i_{s}}\right)=0$ if $i_{1}<p-1$; $h_{p}\left(\lambda_{p-1} \lambda_{i_{2}} \cdots \lambda_{i_{s}}\right)=\lambda_{i_{2}} \cdots \lambda_{i_{s}}$. (See [3] , [5].)

The proofs of the following propositions are straightforward but tedious computations in $\Lambda$. We have omitted the proofs.

Proposition 5.1. $\Lambda(p)_{k} \cdot \Lambda(p+k) \subseteq \Lambda(p)$ (all $\left.k \geqslant 0\right)$.

PROPOSITION 5.2. If $\lambda_{I} \in \Lambda(p)_{k}$ and $\lambda_{J} \in \Lambda(p+k-1)$ then $h_{p}\left(\lambda_{I} \lambda_{J}\right)=$ $h_{p}\left(\lambda_{I}\right) \cdot \lambda_{J}$ in $\Lambda(2 p-1)$.

To state the next result we must first define a map $\Lambda \rightarrow \Lambda$ by sending any admissible $\lambda_{i_{1}} \cdots \lambda_{i_{s}}$ into $\lambda_{2 i_{1}+1} \cdots \lambda_{2 i_{s}+1}$; briefly, $\lambda_{I} \rightarrow \lambda_{2 I+1}$. See [14]. This mapping respects both product and differential.

Proposition 5.3. If $\lambda_{I} \in \Lambda(p)_{k}$ and $\lambda_{J} \in \Lambda(p+k)$ are admissibles, then $h_{p}\left(\lambda_{I} \lambda_{J}\right)=h_{p}\left(\lambda_{I}\right) \cdot \lambda_{J}+\lambda_{2 I+1} h_{p+k}\left(\lambda_{J}\right)$. In particular, the right-hand side of this equation lies in $\Lambda(2 p-1)$.

6. The complexes $L\left(S^{p}\right)$. We can now construct the resolutions $L\left(S^{p}\right)$. Define for each $s \geqslant 0$ an injective unstable $A$-comodule: $L^{s}\left(S^{p}\right)=A \underline{Q}$ $\left(\Lambda(p)^{s} \otimes S^{p}\right)$. Here $\Lambda(p)^{s}$ denotes the graded $Z_{2}$-module $\Sigma_{k \geqslant 0} \oplus \widehat{\Lambda}(p)_{k}^{s}$, and the effect of tensoring over $Z_{2}$ with $S^{p}$ is to raise internal degree by $p$. Define a map of graded vector spaces $\theta: A \rightarrow \Lambda^{1}$ by setting $\theta\left(\xi_{1}^{n_{1}} \cdots \xi_{l}^{n_{l}}\right)=$ $\lambda_{n_{1}-1}$ if $n_{2}=\cdots=n_{l}=0$, and $\theta\left(\xi_{1}^{n_{1}} \cdots \xi_{l}^{n_{l}}\right)=0$ otherwise. Define an $M_{A}$ morphism $d_{\theta}: L^{s}\left(S^{p}\right) \rightarrow L^{s+1}\left(S^{p}\right)$ by giving the associated $\underline{d}_{\theta}: A \underline{\otimes}$ $\left(\Lambda(p)^{s} \otimes S^{p}\right) \rightarrow \Lambda(p)^{s+1} \otimes S^{p}:$

$$
\underline{d}_{\theta}\left(a \otimes \lambda_{I} \otimes \sigma_{p}\right)=\lambda_{I}(\theta a) \otimes \sigma_{p}
$$

Note that if $\lambda_{I} \in \Lambda(p)_{k}^{s}$ then by the definition of $\otimes$ we have $a \in J(p+k)$. Then Proposition 5.1 gives $\lambda_{I}(\theta a) \in \Lambda(p)$, so that (6.1) is meaningful. (It is 
crucial that we have let $A$ act on the right of $\Lambda$ !) Now define a differential $d: L^{s}\left(S^{p}\right) \rightarrow L^{s+1}\left(S^{p}\right)$ by setting $d=A \otimes d_{\Lambda} \otimes S^{p}+d_{\theta} . d$ is an unstable analogue of a "twisted" differential (compare [6]): the verification that $d d=0$ is straightforward.

We must now show the complex $L\left(S^{p}\right)$ acyclic. To this end we extend $h_{p}$ to an $M_{A}$ morphism $h: \Omega L^{s}\left(S^{p}\right) \rightarrow L^{s-1}\left(S^{2 p-1}\right)$ by setting $h\left(\Omega\left(a \otimes \lambda_{I} \otimes \sigma_{p}\right)\right)=a \otimes h_{p}\left(\lambda_{I}\right) \otimes \sigma_{2 p-1}$. Then $h: \Omega L\left(S^{p}\right) \rightarrow L\left(S^{2 p-1}\right)$ is a chain map of homological degree -1 : to verify $h d=d h$ one uses Proposition 5.2. Next we extend the inclusion $\Lambda(p-1) \subset \Lambda(p)$ to a chain map $e: L\left(S^{p-1}\right)$ $\rightarrow \Omega L\left(S^{p}\right)$ of homological degree zero by setting $e\left(a \otimes \lambda_{I} \otimes \sigma_{p-1}\right)=$ $\Omega\left(a \otimes \lambda_{I} \otimes \sigma_{p}\right)$. It is now easy to see that:

Proposition 6.1. The sequence (3.1) is short exact.

Proposition 6.1 is the main tool in proving $L\left(S^{p}\right)$ acyclic. The first step is:

LEMma 6.2. The map $S^{p} \rightarrow L^{0}\left(S^{p}\right)$ given by $\sigma_{p} \rightarrow 1 \otimes \sigma_{p}$ induces an isomorphism $S^{p} \rightarrow H^{0}\left(L\left(S^{p}\right)\right)$.

Proof. Let $A^{+}=\Sigma_{k>0} \oplus A_{k}$. It suffices to show that the composition

$$
A^{+} \stackrel{\psi}{\longrightarrow} A \otimes A \stackrel{A \otimes \theta}{\longrightarrow} A \otimes \Lambda
$$

is one-one. But this is straightforward.

LEMMA 6.3. $H^{1}\left(L\left(S^{p}\right)\right)=0$, and $h: \Omega L\left(S^{p}\right) \rightarrow L\left(S^{2 p-1}\right)$ induces an isomorphism $H^{1}\left(\Omega L\left(S^{p}\right)\right) \rightarrow H^{0}\left(L\left(S^{2 p-1}\right)\right)$.

Proof. By induction on $p$. Cases $p=0,1$ are easy. Suppose the lemma proved for all $p<p_{0}$. From Lemma 6.2 we know $\lambda: H^{0}\left(L\left(S^{p_{0}}\right)\right) \rightarrow H^{0}\left(L\left(S^{p^{0}}\right)\right)$ is zero, so the exactness of the sequence (2.1) shows that $\delta: H^{0}\left(L\left(S^{p_{0}}\right)\right) \rightarrow$ $H^{1}\left(\Omega L\left(S^{p}\right)\right)$ is monic. But the short exact sequence (3.1) shows that

$$
H^{1}\left(L\left(S^{p_{0}-1}\right)\right) \stackrel{e_{\#}}{\longrightarrow} H^{1}\left(\Omega L\left(S^{p_{0}}\right)\right) \stackrel{h_{\#}}{\longrightarrow} H^{0}\left(L\left(S^{2 p_{0}-1}\right)\right)
$$

is exact, and since $H^{1}\left(L\left(S^{p_{0}^{-1}}\right)\right)=0$ by the inductive hypothesis, $h_{\#}$ is monic. Hence, both $\delta$ and $h_{\#}$ are isomorphisms. Now we appeal again to (2.1) and find an exact sequence

$$
H^{0}\left(L\left(S^{p_{0}}\right)\right) \stackrel{\dot{\delta}}{\longrightarrow} H^{1}\left(\Omega L\left(S^{p_{0}}\right)\right) \rightarrow H^{1}\left(L\left(S^{p_{0}}\right)\right) \stackrel{\lambda}{\longrightarrow} H^{1}\left(L\left(S^{p_{0}}\right)\right)
$$

which implies that $\lambda: H^{1}\left(L\left(S^{p_{0}}\right)\right) \rightarrow H^{1}\left(L\left(S^{p_{0}}\right)\right)$ is monic. So Lemma 2.1 gives $H^{1}\left(L\left(S^{p}\right)\right)=0$ and the induction is complete.

Proposition 6.4. $H^{s}\left(L\left(S^{p}\right)\right)=0$, all $s>0$.

Proof. This is now an easy double induction on $s$ and $p$, using Lemma 
6.3, the short exact sequence (3.1) and Proposition 2.4.

Summarizing, we have:

Proposition 6.5. $L\left(S^{p}\right)$ is an injective resolution of $S^{p}$, so that

$$
\operatorname{Coext}^{s}\left(S^{p+k}, S^{p}\right)=H^{s}\left(\Lambda(p)_{k}^{*}\right)
$$

$e: L\left(S^{p-1}\right) \rightarrow \Omega L\left(S^{p}\right)$ is a chain level suspension, and $h: \Omega L\left(S^{p}\right) \rightarrow L\left(S^{2 p-1}\right)$ is a chain level Hopf invariant. The sequence (3.1) is short exact. Under the isomorphism (6.2) the suspension

$$
E: \operatorname{Coext}^{s}\left(S^{p+k-1}, S^{p-1}\right) \rightarrow \operatorname{Coext}^{s}\left(S^{p+k}, S^{p}\right)
$$

is induced by the inclusion $\Lambda(p-1) \rightarrow \Lambda(p)$; the Hopf invariant

$$
H: \operatorname{Coext}^{s}\left(S^{p+k}, S^{p}\right) \rightarrow \operatorname{Coext}^{s-1}\left(S^{p+k-1}, S^{2 p-1}\right)
$$

is induced by $h_{p}: \Lambda(p) \rightarrow \Lambda(2 p-1)$; and

$$
P: \operatorname{Coext}^{s-1}\left(S^{p+k}, S^{2 p-1}\right) \rightarrow \operatorname{Coext}^{s+1}\left(S^{p+k}, S^{p-1}\right)
$$

is the connecting morphism associated with the short exact sequence

$$
0 \rightarrow \Lambda(p-1) \rightarrow \Lambda(p) \stackrel{h_{p}}{\longrightarrow} \Lambda(2 p-1) \rightarrow 0 .
$$

(6.2) appears already in [3], where it is proved by a different method.

Proposition 6.6. Under the isomorphism (6.2) the composition pairing

$$
\operatorname{Coext}^{s}\left(S^{p+k}, S^{p}\right) \otimes \operatorname{Coext}^{r}\left(S^{p+k+l}, S^{p+k}\right) \rightarrow \operatorname{Coext}^{r+s}\left(S^{p+k+l}, S^{p}\right)
$$

is induced by the pairing $\Lambda(p)_{k} \otimes \Lambda(p+k) \rightarrow \Lambda(p)$ (Proposition 5.1).

Proof. Let $\alpha \in \operatorname{Coext}^{s}\left(S^{p+k}, S^{p}\right)$ be given. Suppose it is represented by a cycle $\varphi(\alpha): S^{p+k} \rightarrow L\left(S^{p}\right)$. Since $\varphi(\alpha)$ is an $M_{A}$ morphism we have $\varphi(\alpha)\left(\sigma_{p+k}\right)=1 \otimes \lambda \otimes \sigma_{p}$ for some $\lambda \in \Lambda(p)_{k}$ satisfying $d_{\Lambda}(\lambda)=0$. Then we can extend $\varphi(\alpha)$ to a chain map $\varphi(\alpha): L\left(S^{p+k}\right) \rightarrow L\left(S^{p}\right)$ by setting

$$
\varphi(\alpha)\left(a \otimes \lambda_{I} \otimes \sigma_{p+k}\right)=a \otimes \lambda \lambda_{I} \otimes \sigma_{p}
$$

for any $a \in A, \lambda_{I} \in \Lambda(p+k)$. That $\varphi(\alpha)$ is indeed a chain map follows from our definition (6.1) of $\underline{d}_{\theta}$ : since in (6.1) $A$ is acting on the right of $\Lambda(p+k)$, and in (6.3) $\Lambda(p)_{k}$ is acting on the left of $\Lambda(p+k)$, the two actions commute. From this observation the proposition follows immediately.

We can now go beyond Proposition 3.7 in giving formulas for the Hopf invariant of a composition. Notice first that by means of (6.2), we can use the chain mapping $\lambda_{I} \rightarrow \lambda_{2 I+1}$ to define a homology operation called $S q^{0}$ : $\operatorname{Coext}^{s}\left(S^{p+k}, S^{p}\right) \rightarrow \operatorname{Coext}^{s}\left(S^{2 p+2 k}, S^{2 p}\right)$. Combining Propositions 6.5, 6.6 and 
5.3 we obtain:

Proposition 6.7. If $\alpha \in \operatorname{Coext}^{r}\left(S^{p+k-1}, S^{p-1}\right), \beta \in \operatorname{Coext}^{s}\left(S^{p+k+l}, S^{p+k}\right)$ then $H(E \alpha \cdot \beta)=E S q^{0} \alpha \cdot H \beta$ in $\operatorname{Coext}^{r+s-1}\left(S^{p+k+l-1}, S^{2 p-1}\right)$.

Proposition 6.8. If $\alpha \in \operatorname{Coext}^{r}\left(S^{p+k}, S^{p}\right), \beta \in \operatorname{Coext}^{s}\left(S^{p+k+l}, S^{p+k}\right)$ then $E H(\alpha \cdot \beta)=E H \alpha \cdot \beta+S q^{0} \alpha \cdot E H \beta$ in $\operatorname{Coext}^{r+s-1}\left(S^{p+k+l}, S^{2 p}\right)$.

We remark finally that Propositions 5.1, 5.2, 5.3 can also be used to derive formulas for the Hopf invariant of a Massey product. This we leave to the reader.

\section{BIBLIOGRAPHY}

1. M. G. Barratt, Note on a formula due to Toda, J. London Math. Soc. 36 (1961), 95-96. MR 23 \#A2881; errata 25, 1242.

2. M. G. Barratt and P. J. Hilton, On join operations in homotopy groups, Proc. London Math. Soc. (3) 3 (1953), 430-445. MR 15, 643.

3. A. K. Bousfield and E. B. Curtis, A spectral sequence for the homotopy of nice spaces, Trans. Amer. Math. Soc. 151 (1970), 457-479. MR 42 \#2488.

4. A. K. Bousfield, E. B. Curtis, D. M. Kan, D. G. Quillen, D. L. Rector and J. W. Schlesinger, The mod-p lower central series and the Adams spectral sequence, Topology 5 (1966), 331-342. MR 33 \#8002.

5. E. B. Curtis, Simplicial homotopy theory, Advances in Math. 6 (1971), 107-209. MR 43 \#5529.

6. V. K. A. M. Gugenheim, On a theorem of E. H. Brown, Illinois J. Math. 4 (1960), 292-311. MR 22 \#2990.

7. P. J. Hilton, On the Hopf invariant of a composition element, J. London Math. Soc. 29 (1954), 165-171. MR 15, 734.

8. D. Kraines, On excess in the Milnor basis, Bull. London Math. Soc. 3 (1971), 363365. MR 45 \#9317.

9. W. S. Massey and F. P. Peterson, The mod 2 cohomology structure of certain fiber spaces, Mem. Amer. Math. Soc. No. 74 (1967). MR 37 \#2226.

10. J. W. Milnor, The Steenrod algebra and its dual, Ann. of Math. (2) 67 (1958), 150-171. MR 20 \#6092.

11. N. E. Steenrod, Cohomology operations, revised by D. B. A. Epstein, Ann. of Math. Studies, no. 50, Princeton Univ. Press, Princeton, N. J., 1962. MR 26 \#3056.

12. H. Toda, Composition methods in homotopy groups of spheres, Ann. of Math. Studies, no. 49, Princeton Univ. Press, Princeton, N. J., 1962. MR 26 \#77.

13. - Reduced join and Whitehead product, J. Inst. Polytech. Osaka City Univ. Ser. A 8 (1957), 15-30. MR 19, 159.

14. J. S. P. Wang, On the cohomology of the mod-2 Steenrod algebra and the nonexistence of elements of Hopf invariant one, Illinois J. Math. 11 (1967), 480-490. MR 35 \#4917.

DEPARTMENT OF MATHEMATICS, BOSTON COLLEGE, CHESTNUT HILL, MASSACHUSETTS 02167

Current address: Department of Mathematics, Fordham University, Bronx, New York 10458 